\title{
Jugend, Gesundheit, Lebensstil. Entstehung und Konzeption des HBSC-Projekts
}

\author{
Adolescence, Health, Lifestyle. Development and Philosophy of the HBSC Project
}

Ziemlich genau 30 Jahre ist es her, dass sich Jugend- und Gesundheitsforscher zuerst aus Großbritannien, Finnland und Norwegen, dann auch aus Dänemark und Österreich zusammen setzten und die Vereinbarung trafen, international vergleichende Forschung zur Jugendgesundheit einzuleiten. Sie störte das Defizit an wissenschaftlich abgesicherten Informationen und Daten, das sie in ihren Ländern verzeichneten, aber mehr noch das Problem, diese Daten international nicht miteinander vergleichen zu können. Jedes Land hatte zwar seine eigene Forschungstradition in diesem Bereich, aber Erhebungstechnik und Methodik wichen so deutlich voneinander $\mathrm{ab}$, dass keine Quervergleiche möglich waren. Früh wandten sich die beteiligten Wissenschaftlerinnen und Wissenschaftler an die Weltgesundheitsorganisation mit ihrem Regionalbüro in Kopenhagen. Dort wurde die Initiative begrüßt und fortan mit in das Arbeitsprogramm aufgenommen. Das international vergleichende Forschungsvorhaben „Health Behaviour in schoolaged Children (HBSC)“ war geboren [1]. Es dauerte noch 10 Jahre, bis der Initiatorenkreis an mich herantrat und fragte, ob ich als Principal Investigator für Deutschland fungieren wolle. Seinerzeit lief in Bielefeld der interdisziplinäre Sonderforschungsbereich „Prävention und Intervention im Kindes- und Jugendalter“, der mehrere international vergleichende Vorhaben auch zur Jugendgesundheit umfasste. Da 1993 auch die erste deutsche School of Public Health, die Fakultät für Gesundheitswissenschaften, an der Universität Bielefeld gegründet worden war und ich als erster Dekan fungierte, war der Standort Bielefeld geradezu prädestiniert, die Koordinierungsrolle für Deutschland zu übernehmen.

Das Konzept des Jugendgesundheits-Surveys HBSC passte nahtlos in die theoretische Ausrichtung sowohl des Sonderforschungsbereiches als auch der Forschungsgruppen an der Fakultät für Gesundheitswissenschaften. Die Kernidee war und ist, das Gesundheitsverhalten und das Gesundheitsbewusstsein von Jugendlichen in den ersten Jahren der Pubertät systematisch zu erforschen und bei der theoretischen Analyse interdisziplinäre Ansätze zu verwenden. In Bielefeld hatten wir zur damaligen Zeit den Begriff „Gesundheit" gerade neu konzipiert. Auf den Spuren der Weltgesundheitsorganisation hatten wir ihn aus den engen Bezügen des spezialisierten Krankheitsversorgungssystems gelöst. Der neue Gesundheitsbegriff betonte - im Gegensatz zum bis dahin vorherrschenden Ansatz - die Verankerung von Wohlbefinden in allen Dimensionen des täglichen Lebens. Gesundheit ist demnach beeinträchtigt, wenn sich in einem oder mehreren dieser Bereiche Anforderungen ergeben, die von der Person in der jeweiligen Phase im Lebenslauf nicht erfüllt und bewältigt werden können. Die Beeinträchtigung kann sich, muss sich aber nicht, in Symptomen der sozialen, psychischen und physisch-physiologischen Auffälligkeit manifestieren [2,3].

Das internationale HBSC-Forschungsteam ging von der gleichen Gesundheitsdefinition aus. Das Gesundheitsverhalten von Jugendlichen wurde im sozialen ebenso wie im biologischen Kontext thematisiert. Das Gesundheitsverhalten Jugendlicher wurde als Teil ihres Lebensstils konzipiert, und dieser Lebensstil wiederum wurde ganz stark im Zusammenhang mit den objektiven sozialen Lebensbedingungen als Determinanten von Gesundheit und Krankheit wahrgenommen. Diese Schwerpunkte sind bis heute die Orientierungspunkte für die international vergleichenden Untersuchungen geblieben [4]. Der klugen Weichenstellung des Initiatorenteams zu Beginn der 1980er Jahre kann man also nur größten Respekt bezeugen.

Für Deutschland brachte die Beteiligung von der ersten Stunde an die Chance, an international abgestimmten Untersuchungen teilzunehmen und damit schon in den 1990er Jahren die Voraussetzungen dafür zu schaffen, forscherische Kapazitäten und finanzielle Möglichkeiten für Untersuchungen von überregionaler Bedeutung einzuleiten. Die Beteiligung am HBSC-Forschungsteam wirkte aber auch nach innen, indem die zunächst nur auf das größte Bundesland Nordrhein-Westfalen bezogenen Jugendgesundheitsstudien nach dem internationalen Forschungsprotokoll im Laufe der Jahre auf immer mehr Bundesländer ausgedehnt wurden. Durch das Team in Bremen (Petra Kolip), Berlin und Hamburg (Ulrike Ravens-Sieberer), Dresden (Wolfgang Melzer), Halle (Mathias Richter) und Frankfurt (Andreas Klocke) ist inzwischen innerhalb der Bundesrepublik Deutschland im Rahmen der des HBSC-Konsortiums eine Erfassung gesundheitlich relevanter Daten für Jugendliche der Altersgruppe 11, 13 und 15 Jahre möglich geworden, mittels derer systematische regionale Vergleiche erfolgen können. Die Untersuchungen werden im Rhythmus von 4 Jahren wiederholt, sodass zuverlässige und mit sicherer Methodik abgesicherte Zeitreihen gebildet werden können. Auf diese Weise stehen auch für die innerdeutsche Diskussion in Theorie und Praxis Daten für Trendanalysen zum Gesundheitsverhalten, Gesundheitsbewusstsein und subjektiven Gesundheitsstatus dieser Altersgruppe zur Verfügung. Sie liefern zuverlässige 
und belastbare Informationen für die Jugendgesundheitspolitik und die Gesundheitsförderung in verschiedensten Bereichen. Eine ganze Reihe von präventiven Strategien konnten in den letzten Jahren im Zusammenhang mit der Interpretation der Daten von HBSC Deutschland entwickelt und systematisch überprüft werden. So konnte beispielsweise die Tabak- und Alkoholpolitik in Deutschland in die öffentliche Diskussion gerückt werden, was schließlich dazu führte, dass der Konsum von Alkopops deutlich abgesunken ist, und der Tabakkonsum auf einem historisch bisher nie dagewesenen Tiefstand ist.

An diesen Beispielen lässt sich erkennen, wie eine nach strengen methodischen Kriterien durchgeführte, regelmäßig wiederholte und damit Trendaussagen ermöglichende und internationale Referenzen herstellende wissenschaftliche Forschung auf Dauer nicht nur wissenschaftliche, sondern auch praktische Effekte im Bereich des Gesundheitsverhaltens erzielen kann. Neben dem Tabak- und Alkoholkonsum sind inzwischen auch alle anderen in den HBSC-Studien erfassten Gesundheitsindikatoren mit in diese Diskussion eingegangen. Auch zu geschlechtsspezifischen Mustern in Gesundheitsverhalten und Gesundheitswahrnehmung ist im Laufe der letzten 10 Jahre bereits so viel an Erkenntnissen gewonnen worden, dass erste effiziente Präventions- und Förderstrategien entwickelt wurden. Diese Ergebnisse werden in Praxis und Politik immer stärker herangezogen, um geschlechtssensible Präventionsstrategien und Programme der Gesundheitsförderung zu entwickeln.
Insgesamt ist HBSC International und HBSC Deutschland also eine wissenschaftliche Erfolgsgeschichte mit Ausstrahlung auf den Bereich von Prävention und Gesundheitsförderung. Die in diesem Themenheft versammelten Beiträge der heute in Deutschland tätigen Forschungsteams und des HBSC-Konsortiums bezeugen den erreichten hohen methodischen und theoretischen Forschungsstand. Meinen beiden Nachfolgerinnen in der Funktion des Principal Investigators für Deutschland und der Leitung des WHO Collaborating Center for Child and Adolescent Health Promotion - Prof. Ulrike Ravens-Sieberer und Prof. Petra Kolip - möchte ich deswegen nachdrücklich für ihre engagierte Arbeit danken, die auch dieses Themenheft möglich gemacht hat.

\section{Literatur}

1 Currie C, Gabhainn SN, Godeau E. International HBSC Network Coordinating Committee. The Health Behaviour in School-aged Children: WHO Collaborative Cross-National (HSBC) Study: origins, concept, history and development 1982-2008. International Journal of Public Health 2009; 54: 131-139

2 Hurrelmann K. Sozialisation und Gesundheit. Weinheim: Juventa; 1988

3 Hurrelmann K. Human Development and Health. New York: Springer; 1989

4 Ottova V, Ravens-Sieberer $U$. Social determinants in Child health: reflections from the Health Behaviour in School-aged Children survey. International Journal of Public Health 2010; 55: 525-526 